\title{
Combined White Matter Imaging Suggests Myelination Defects in Visual Processing Regions in Schizophrenia
}

\author{
Lena Palaniyappan*, ${ }^{*, 2}$, Ali Al-Radaideh ${ }^{3}$, Olivier Mougin ${ }^{4}$, Penny Gowland ${ }^{4}$ and Peter F Liddle' \\ 'Division of Psychiatry, Institute of Mental Health, University of Nottingham, Nottingham, UK; ${ }^{2}$ Nottinghamshire Healthcare NHS Trust, \\ Nottingham, UK; ${ }^{3}$ Department of Medical Imaging, Faculty of Allied Health Sciences, The Hashemite University, Zarqa, Jordan; ${ }^{4}$ Sir Peter \\ Mansfield MR Centre, University of Nottingham, Nottingham, UK
}

\begin{abstract}
Diverse pathological changes occur in the white matter (WM) of patients with schizophrenia. Various microstructural alterations including a reduction in axonal number or diameter, reduced myelination, or poor coherence of fibers could account for these changes. Abnormal integrity of macromolecules such as myelin ('dysmyelination') can be studied by applying multiple modalities of WM imaging such as diffusion tensor imaging (DTI) and magnetization transfer imaging (MTI) in parallel. Using ultra-high field (7 Tesla) MTI in I7 clinically stable patients with schizophrenia and 20 controls, we evaluated the voxelwise distribution of macromolecular WM abnormalities. Patients had a significant reduction in magnetization transfer ratio (MTR) in WM adjacent to visual processing regions and inferior temporal cortex (Cohen's $d=1.54$ ). Among the regions showing MTR reduction, a concurrent reduction in fractional anisotropy (FA) occurs proximal to the lingual gyrus. Multiple regression analysis revealed that the degree of FA reduction in the putatively 'dysmyelinated' regions in patients predicted impaired processing speed (PS; $\beta=0.74 ; P=0.003$ ), a core cognitive dysfunction in schizophrenia. In controls, MTR/FA in the occipito-temporal regions were not associated with PS. Our findings suggest that dysmyelination in visual processing regions is present in patients with schizophrenia with greatest cognitive and functional impairment. Combined DTI/MTI deficits in the occipito-temporal region may be an important variable when considering potential treatment targets for improving cognitive function in schizophrenia.
\end{abstract}

Neuropsychopharmacology (2013) 38, I808-18I5; doi:I0.1038/npp.2013.80; published online 8 May 20।3

Keywords: myelination; magnetization transfer; visual processing; occipital; schizophrenia

\section{INTRODUCTION}

Pathophysiological processes affecting the formation of myelin has been a focus of attention in schizophrenia for the last 3 decades (Walterfang et al, 2006). Despite the scarcity of neuropathological studies, several lines of evidence from neuroimaging, immunological, and genetic investigations implicate a dysfunction of the myelination process. Conduction delay associated with aberrant myelination is seen as a central feature of various mechanistic models of psychosis (Whitford et al, 2012). Diffusion tensor imaging (DTI) studies suggest a role for WM pathology in the cognitive deficits (Pérez-Iglesias et al, 2010), with a large body of evidence implicating a role for myelin deficits in cognitive dysfunction seen in schizophrenia (Voineskos et al, 2012a). In particular, observations from healthy controls (Bartzokis et al, 2010) suggest that interindividual

\footnotetext{
*Correspondence: Dr L Palaniyappan, Division of Psychiatry, Institute of Mental Health Building, University of Nottingham, Room-09, C Floor, Triumph Road, Nottingham, NG7 2TU, UK, Tel: + 44 II5 823 0407, Fax: + 44 II5 8230433 ,

E-mail: Lena.Palaniyappan@nottingham.ac.uk

Received 3 December 2012; revised 26 February 2013; accepted 26 March 2013; accepted article preview online 4 April 2013
}

variability in task performance for crucial cognitive measures, such as processing speed (PS), depend on the integrity of myelination. Despite this intuitive postulation of the role of myelin defects in schizophrenia, lack of reliable in vivo approaches to measure myelin-related WM abnormalities has impeded progress in this field. Magnetization transfer imaging (MTI) provides a promising tool for studying putative myelin defects (Chen et al, 2007). Magnetization transfer ratio (MTR) represents the exchange of magnetization between protons bound to macromolecules such as myelin and free water. In demyelination disorders such as MS, the quantification of MTR is considered as a reliable marker of the burden of myelin damage, and used as a surrogate marker of therapeutic efficacy (Ge et al, 2001). In schizophrenia, MTR abnormalities have been previously demonstrated in temporooccipital and frontal regions (Walterfang et al, 2006).

Both DTI and MTI are nonspecific techniques that can be affected by a range of pathological changes in brain tissue. As a result, the inferences drawn from studies that use either DTI or MTI as stand-alone imaging techniques are limited by this lack of specificity. MT quantifies a purely scalar measure that reflects tissue composition (especially myelin content) more than the spatial organization of WM 
tracts (Schmierer et al, 2004). FA appears to be influenced primarily by microstructural properties, such as fiber count, volume, and directionality (Stikov et al, 2011), but is also affected by myelin content (Schmierer et al, 2007). Therefore, while isolated MTR reduction (without FA changes) can be considered as a reflection of reduced myelin content, the presence of combined FA and MTR reduction in a region is strongly suggestive of pathology affecting myelinated fibers. Kubicki et al (2005) proposed that in schizophrenia, combined WM abnormalities observed in both DTI and MTI are likely to reflect more specifically the abnormalities in the microstructural properties of myelinated axons.

The magnetization transfer phenomenon is particularly well captured at ultra-high field (7T) MR imaging (Mougin et al, 2010), with a higher sensitivity to detect macromolecular tissue composition. In the present study, we employ 7T MR for the first time in schizophrenia, and use a voxel-based approach to locate brain regions showing significant MTR changes in patients. We also employ DTI and structural imaging at 3T strength in the same sample of subjects to study changes in the FA and WM volume in brain regions showing MTR abnormalities. From prior MTI studies in schizophrenia, we expected widespread reduction in the MTR in patients involving both frontal and occipitotemporal WM.

Given the association between WM pathology and PS deficits (Bartzokis et al, 2010; Turken et al, 2008; Vernooij, 2009), we investigated which of the three WM measures (WM volume, FA, or MTR) in the affected brain regions predicted performance in a digit-symbol substitution test (DSST) in patients. In particular, occipito-temporal projections appear to facilitate the processing of visual information (Catani et al, 2003). DTI studies in healthy individuals report that the age-related disruption of the occipitotemporal projections predicts performance in tasks that require high visuomotor PS (Voineskos et al, 2012b; Zahr et al, 2009). In light of these observations, we expected that the abnormal occipito-temporal WM integrity in patients would predict PS deficits.

\section{MATERIALS AND METHODS}

Twenty patients with schizophrenia and 21 healthy controls were originally recruited, of whom the data from 17 patients and 20 controls are reported here, after exclusion of cases with excessive movement, as described below. Patients aged 18-55 years with a DSM-IV (American Psychiatric Association, 1994) diagnosis of schizophrenia were included. Diagnosis was ascertained using a consensus procedure (Leckman et al, 1982) after reviewing clinical notes, collecting information from the psychiatrists providing clinical care, and conducting a structured diagnostic interview (Signs and Symptoms in Psychotic Illness (Liddle et al, 2002)) with the patients. All patients were in a stable phase of illness (defined as a change of no more than 10 points in their Global Assessment of Function (GAF, DSMIV (American Psychiatric Association, 1994)) score, assessed 6 weeks before and immediately before study participation). The mean duration of illness was 7 years $(\mathrm{SD}=7.9)$. Subjects with age $<18$ or $>55$ years, subjects with neurological disorders, current substance dependence, or IQ $<70$ using Quick Test (Ammons and Ammons, 1962) were excluded. Twenty-one healthy subjects group matched for age, gender, and parental socioeconomic status with the patient group were recruited from the local communities through advertisements. In addition to the exclusion criteria specified for patients, controls were excluded if there was a personal or family history of psychosis. All subjects were recruited from Nottinghamshire, UK.

Handedness was assessed using the 12-items Annett scale (Annett, 1970). The median defined daily dose (DDD) of antipsychotics(WHO Collaborating Centre for Drug Statistics and Methodology, 2003) was calculated for all patients. All subjects were interviewed on the same day as the scan, and symptom scores assigned according to the SSPI for both patients and controls. Written and oral versions of the DSST, similar in format to the DSST from the Wechsler Adult Intelligence Scale (Wechsler, 1997), were also administered. The score was the number of symbols correctly assigned within a period of $90 \mathrm{~s}$. For each subject, a mean DSST score was computed from the two formats.

Permission for the study was obtained from Nottinghamshire research ethics committees. All participants gave written informed consent.

\section{MTI Acquisition}

Scanning was performed on a 7T Philips Achieva system with 32-channel receive coil. MT images were acquired using a 3D magnetization transfer-prepared turbo field echo (MTTFE) sequence in two volumes (A no saturation pulse, B $-1.05 \mathrm{kHz}$ resonance saturation pulse (sensitive to magnetization and chemical exchange saturation transfer effects)) with $1 \times 1 \times 1 \mathrm{~mm}$ voxel size; field of view $(\mathrm{FOV})=200 \times 169 \times 74$; echo time $(\mathrm{TE})=5.8 \mathrm{~ms}$; repetition time $(\mathrm{TR})=10.2 \mathrm{~ms}$; flip angle $=8^{\circ} ; 74$ slices; total scan time $=9 \mathrm{~min}$.

\section{MPRAGE Acquisition}

7T T1-weighted images were acquired using a 3D MTTFE (IR-TFE) with $0.6 \times 0.6 \times 0.6 \mathrm{~mm}^{3}$ voxel size; $\mathrm{FOV}=192$ $\times 180 \times 140 \mathrm{~mm} ; \mathrm{TE}=5.6 \mathrm{~ms} ; \mathrm{TR}=15 \mathrm{~ms}$; flip angle of the TFE readout pulse $=8^{\circ} ; 260$ slices; TFE factor per inversion $=148$; inversion time $=1175 \mathrm{~ms}$; shot-to-shot interval $=3000 \mathrm{~ms}$; total scan time $=12 \mathrm{~min}$.

\section{DTI Acquisition}

For DTI, we chose to use 3T field strength (Philips Achieva) rather than the $7 \mathrm{~T}$, as the gradient performance (a limiting factor for DTI acquisition) is similar at 7T and 3T. At higher field strength, although the signal-to-noise ratio improves, T2 is shortened, annulling any specific advantage for DTI expected when scanning at 7T field (Polders et al, 2009; Speck and Zhong, 2009). We also noted that eddy currents (an important source of image distortion in DTI) were more pronounced at $7 \mathrm{~T}$ than at $3 \mathrm{~T}$.

Diffusion-weighted images were acquired using a singleshot, spin-echo, echo planar imaging (EPI) sequence in alignment with the anterior commissure-posterior 
commissure plane. The acquisition parameters were as follows: TE, $56.9 \mathrm{~ms}$; TR, $8.63 \mathrm{~s} ; 112 \times 112$ matrix; voxel size $=2 \mathrm{~mm}$ isotropic; $\mathrm{FOV}=224 \times 224 \times 104 ; \mathrm{TE}=56.9$ $\mathrm{ms} ; \mathrm{TR}=8.63 \mathrm{~s}$; flip angle $=90^{\circ} ; 52$ slices; 32 directions with b-factor of $1000 \mathrm{~s} / \mathrm{mm}^{2}$; EPI factor $=59$; total scan time $=6.29 \mathrm{~min}$.

All scans were inspected immediately after each acquisition, and if motion was detected the scans were repeated. MTI images from one control subject and three patients could not be included in the study owing to significant movement-induced signal changes. In addition, one patient had significant movement artifacts in DTI images. A total of 20 controls and 17 patients were included in the MTI analysis; 20 controls and 16 patients were included in the DTI analysis. The excluded patients had similar clinical and demographic profile to the subjects who were included in the final analysis. (Mean (SD) values for excluded subjects: age $=39.3(10.1)$ years; duration of illness $=13.3(8.5)$ years, antipsychotics $\mathrm{DDD}=1.1(0.4) ; \mathrm{GAF}=46.67(4.2))$. The $3 \mathrm{~T}$ and 7T scans were done one after the other in the same order (3T followed by $7 \mathrm{~T}$ ) on the same day for all subjects, with $5-10 \mathrm{~min}$ of time interval between the scans for transferring the patients between two scanners located in the same building.

\section{Data Preprocessing}

Diffusion-weighted images were converted from DICOM to analyze images. Using FMRIB's Diffusion Toolbox in FMRIB Software Library (FSL) (http://www.fmrib.ox.ac.uk/fsl), differences in spatial distortion due to eddy currents were corrected. A binary mask of the brain was then generated using Brain Extraction tool in FSL. Diffusion tensor and associated parameters such as fractional anisotropy (FA), which measures the deviation from isotropic diffusion, were then calculated using the DTIFIT tool in FSL. The nondiffusion-weighted volume (b-value, a function of diffusion gradient strength and time interval $=0$ ) was co-registered to the $7 \mathrm{~T} \mathrm{~T} 1$ image with 12 degrees of freedom using FLIRT linear registration algorithm, using the 'mutual information' cost function with low number (100) of bins and trilinear interpolation. The registration matrix was later applied to FA maps to transfer them into the same space of the T1 image.

For each voxel in the MTI images, magnetization transfer ratio was calculated on a pixel-by-pixel basis by the formula $\mathrm{MTR}=\{(\mathrm{Mo}-\mathrm{Ms}) / \mathrm{Mo}\} \times 100$ percent units, where Mo and Ms are the mean signal intensities without and with the saturation pulse, respectively. The reference volume in the MT sequence was co-registered to the 7T T1-weighted image with six degrees of freedom using FLIRT linear registration algorithm from FSL. Owing to the difference of contrast between the T1 image and reference volume in the MT sequence, again the 'mutual information' cost function and trilinear interpolation method was used. The registration matrix was applied to MTR maps to transfer them into the same space of the T1 image.

Preprocessing using SPM8 was carried out for T1, MTR, and the FA images, using identical procedures to ensure that the images from all modalities had voxel-to-voxel spatial correspondence. Using the SPM8 Diffeomorphic Anatomical Registration Through Exponentiated Lie algebra (Ashburner, 2007) algorithm, grey, white, and CSF tissue were segmented. GM and WM images were separately warped onto group average template. For MTR images, modulation was carried out to account for tissue distortion during template warping; this step was not applied for FA images. The resultant images were resampled to isotropic $3 \mathrm{~mm}$ voxels, normalized to MNI space, and smoothed using a Gaussian kernel of $8 \mathrm{~mm}$ full width half-maximum to enable voxel-based morphometric comparison of the two groups. Total brain volume was computed from T1 images using the sum of the volumes of GM, WM, and CSF segments.

\section{Statistical Analysis}

The MTR maps of the two groups were compared using the General Linear Model framework implemented in SPM8. A two-sample $t$ tests using a voxelwise $P<0.001$ and a clusterlevel familywise error correction at $P=0.05$ was carried out, with age and gender as covariates for MTR and FA analysis. The FA analysis was constrained within a mask obtained from the group comparison contrast of MTR maps at an uncorrected threshold of $P<0.001$. Principal eigenvariate representing the typical MTR and FA from the voxels included within this mask (in each case, explaining $>85 \%$ variance) were extracted and used in a multiple regression analysis predicting the mean DSST scores separately for controls and patients with age, gender, and total WM volume as covariates. All variables (covariates and predictors) were entered in a single step in the regression model.

\section{RESULTS}

\section{Clinical and Demographic Variables}

The demographic and clinical characteristics of the sample are presented in Table 1. Patients did not differ from the controls in terms of age, gender, handedness, or parental

Table I Clinical and Demographic Features

\begin{tabular}{|c|c|c|}
\hline Features & $\begin{array}{c}\text { Patients }(N=I 7) \\
\text { Mean/n }(S D)\end{array}$ & $\begin{array}{c}\text { Controls }(N=20) \\
\text { Mean/n }(S D)\end{array}$ \\
\hline Gender (male/female) & $12 / 5$ & $15 / 5$ \\
\hline Handedness (right/left) & $16 / 1$ & $18 / 2$ \\
\hline Age & $33(10.0)$ & $32(8.2)$ \\
\hline Parental NS-SEC & $2.6(1.7)$ & $2.5(1.6)$ \\
\hline SSPI total score ${ }^{a}$ & $1 \mid .5(\mid 0.1)$ & $0.6(0.8)$ \\
\hline Reality distortion ${ }^{a}$ & $2.5(2.9)$ & $0(0)$ \\
\hline Disorganization ${ }^{\mathrm{a}}$ & $1.2(1.7)$ & $0.1(0.3)$ \\
\hline Psychomotor poverty ${ }^{a}$ & $2.8(4.0)$ & $0(0)$ \\
\hline Illness duration & $7.0(7.9)$ & - \\
\hline DDD of antipsychotics & $0.8(0.7)$ & - \\
\hline GAF score ${ }^{a}$ & $46.9(11.4)$ & $88.1(7.4)$ \\
\hline Mean DSST ${ }^{\mathrm{a}}$ & 44.1 (9.8) & $63.1(12.8)$ \\
\hline
\end{tabular}

Abbreviations: DDD, defined daily dose; DSST, digit-symbol substitution test; GAF, Global Assessment of Functioning; NS-SEC, National Statistics Socio-economic Classification; SSPI, Signs and Symptoms of Psychotic IIIness. a Significantly different between the two groups using unpaired $t$ test $(P<0.05)$. 
SES. Patients had a mean current symptom burden of 11.5 units $(S D=10.1$; range $1-25)$, measured using the SSPI (out of a maximum possible score of 80). As expected, patients had significantly lower scores on the DSST (mean (SD) in patients $=44.1(9.8)$, controls $=63.1(12.8)$, $t(35)=5.0, P<0.001)$ and GAF (mean $(\mathrm{SD})$ in patients $=$ $46.9(11.4)$, controls $=88.1(7.4), t(35)=13.23, P<0.001)$.

\section{MTR Group Differences}

Patients showed a significant reduction in MTR in occipital and temporal WM regions corresponding to inferior longitudinal fasciculus (ILF) bilaterally, right inferior fronto-occipital fasciculus and left anterior thalamic radiation (Table 2 and Figure 1). The effect size of difference estimated from all voxels showing MTR reduction was
1.54 (mean MTR (SD) in patients $=20.91 \% \quad(7.26 \%)$; controls $=29.74 \%(4.03 \%))$.

\section{FA Differences in Regions with MTR Abnormalities}

Within the regions showing MTR reductions in schizophrenia, most significant reduction in FA was located in a cluster (MNI coordinates: $-15,-81,-12$; peak $t=4.08$, $k=16$, FWE corrected $P=0.026)$ that included both left ILF and inferior fronto-occipital fasciculus. Mean FA obtained from all voxels within the mask in patients did not differ significantly from controls (mean FA (SD) in patients $=0.45(0.05)$ controls $=0.47(0.05) t=1.32 P=0.19)$. A whole brain, unconstrained search for FA changes (FWE corrected $P<0.05)$ revealed no significant changes in brain regions outside the mask.

Table 2 MTR Abnormalities in Schizophrenia

\begin{tabular}{|c|c|c|c|c|c|}
\hline Change in patients & $\begin{array}{c}\text { MNI coordinates } \\
\text { of centroid } \\
(x, y, z)\end{array}$ & $\begin{array}{l}\text { Proximal GM } \\
\text { region (AAL atlas) }\end{array}$ & $\begin{array}{l}\text { WM tracts } \\
\text { crossing the } \\
\text { cancroids }\end{array}$ & $\begin{array}{l}\text { Peak } T \\
\text { intensity }\end{array}$ & $\begin{array}{l}\text { Cluster extent } \\
\text { (no. of voxels) }\end{array}$ \\
\hline \multirow[t]{3}{*}{ Reduced MTR } & $15,-93,-6$ & $\begin{array}{l}\text { Right calcarine, } \\
\text { lingual gyrus, } \\
\text { fusiform gyrus }\end{array}$ & $\begin{array}{l}\text { Right inferior fronto-occipital fasciculus } \\
\text { Right ILF }\end{array}$ & 5.03 & 168 \\
\hline & $-48,-21,-27$ & Left inferior temporal gyrus & Left ILF & 3.86 & 216 \\
\hline & $-3,-24,-12$ & Midbrain & Left anterior thalamic radiation & 3.68 & 309 \\
\hline Increased MTR & & None & & & \\
\hline
\end{tabular}

Abbreviation: AAL, Automated Anatomical Labeling Atlas (Tzourio-Mazoyer et al, 2002).

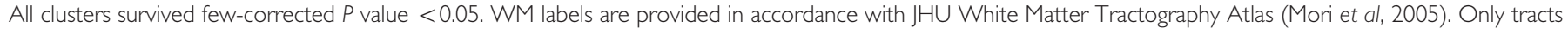
with $>5 \%$ probability are included in the labels.
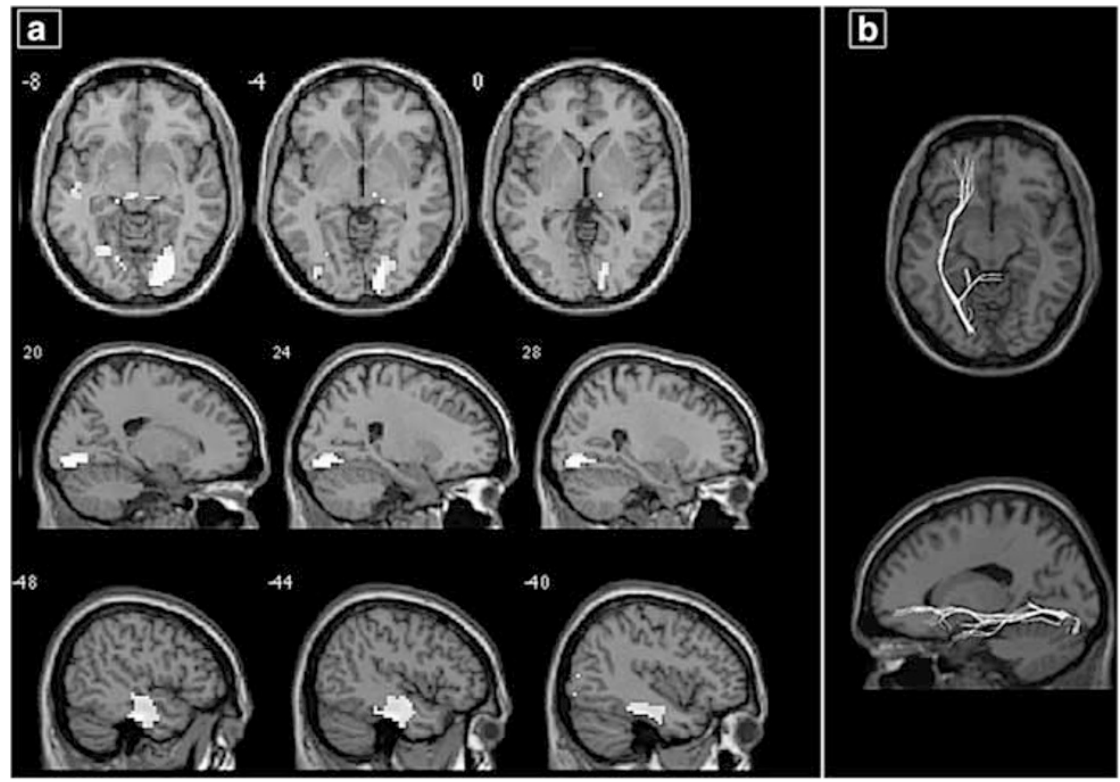

Figure I WM MTR reduction in patients with schizophrenia compared with healthy controls. Illustrations drawn on a single subject structural image (using xjview: http://www.alivelearn.net/xjview8/) with slices selected for the best display of regions showing differences in the two sample $t$ test. (a) displays group differences from voxelwise analysis. (b) displays WM diffusion tensor tracts traversing a rectangular volume centered at the region, showing the most significant combined MTR/FA reduction in patients $(x=-15, y=-81, z=-12$ ), mapped using DTlquery (http://www.graphics.stanford.edu/projects/dti) in a single normal individual. Tracts include the inferior occipito-frontal and the ILF (yellow), on axial and sagittal slices from a structural TI image for illustrative purposes. A full color version of this figure is available at the Neuropsychopharmacology journal online. 


\section{Predicting PS}

The results of the regression analysis predicting DSST scores in the two groups are shown in Table 3. In controls, none of the variables significantly predicted the DSST score. In patients, higher FA in regions showing reduced MTR was associated with higher DSST scores (Figure 2). MTR values did not significantly predict the DSST scores in both groups. In patients, even when antipsychotic dose was added as a predictor variable to the model, FA continued to show a strong association with mean DSST $(\beta=0.74, t=3.53$, $P=0.005)$. Antipsychotic dose itself was not a significant predictor of DSST scores $(\beta=0.007, t=0.03, P=0.9)$.

It is possible that the relationship between FA reduction and PS is not specific to the regions showing MTR changes but also occurs elsewhere in the brain (ie, in regions showing no MTR changes). Therefore, we repeated the regression analysis after simultaneously including the eigenvariate of FA and MTR obtained from regions outside

Table 3 PS, MTR, and FA in Patients and Controls

\begin{tabular}{|c|c|c|c|c|c|c|}
\hline \multirow[t]{2}{*}{ Independent variables } & \multicolumn{3}{|c|}{$\begin{array}{l}\text { Patients }(N=I 7) \\
\quad\left(R^{2}=0.485\right)\end{array}$} & \multicolumn{3}{|c|}{$\begin{array}{c}\text { Controls }(N=20) \\
\quad\left(R^{2}=0.258\right)\end{array}$} \\
\hline & $\beta$ & $t$ & $P$ & $\beta$ & $t$ & $P$ \\
\hline MTR & -0.31 & -1.6 & 0.14 & -0.16 & -0.64 & 0.53 \\
\hline FA & 0.74 & 3.7 & 0.003 & 0.21 & 0.48 & 0.64 \\
\hline Age & 0.02 & 0.11 & 0.91 & -0.44 & -1.82 & 0.09 \\
\hline Gender & 0.13 & 0.62 & 0.55 & 0.54 & 1.63 & 0.13 \\
\hline Total WM volume & 0.19 & 0.89 & 0.39 & 0.46 & 0.91 & 0.38 \\
\hline
\end{tabular}

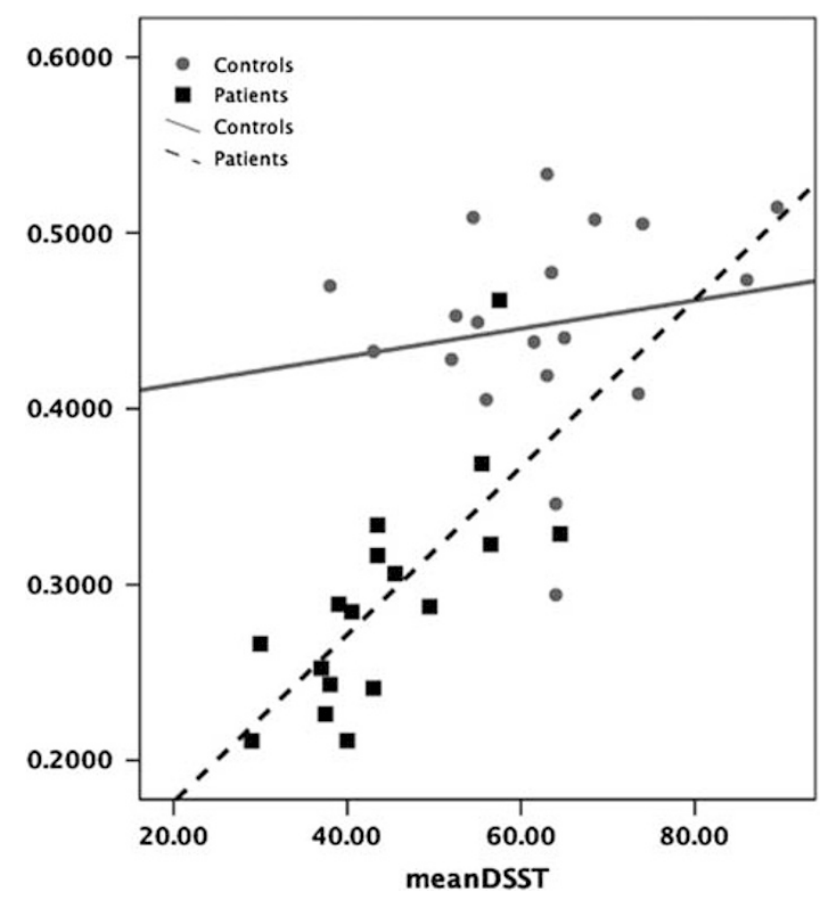

Figure 2 Correlation plot depicting the relationship in both patients and controls between mean DSST scores and the principal eigenvariate of FA within the regions showing MTR reduction in schizophrenia. ('exclusive') of the mask showing MTR changes in schizophrenia, along with age, gender, total WM volume, and within-mask eigenvariates of FA and MTR. In patients, PS continued to show a strong relationship $(t=2.66$, $P=0.026)$ with within-mask FA, and no relationship with FA $(t=1.02, P=0.34)$ or MTR $(t=0.85, P=42)$ outside the mask. Controls continued to show no relationship between PS and the predictors.

There was no indication for multicollinearity of predictors. A positive correlation was observed between the principal eigenvariates of the FA and MTR measures within the mask, although reaching statistical significance only in the control group (Pearson's $r(p)$ in controls $=0.50$ $(P=0.03)$; patients $=0.2(0.44))$. The difference between the two $r$ values was not statistically significant (Fisher's $r$-to- $Z$ transformation $Z=0.95, P=0.34$ ).

Mean MTR and FA values in patients did not show any significant correlation with current antipsychotic dose in DDD or a measure of dose-year approximation (illness duration X present antipsychotic dose; all $P>0.3$ ). Dropping the three left-handed subjects (one patient and two controls) did not affect the distribution of MTR or FA differences between groups.

We also observed MTR reductions in GM adjacent to the WM regions showing significant MTR abnormalities. The GM-MTR results are presented in the Supplementary Material S1.

\section{DISCUSSION}

Using a combined white matter (WM) imaging approach and high-field MT acquisition, this study provides the first evidence for the presence of prominent deficits in macromolecular composition, suggestive of dysmyelination in association with a reduction in axonal integrity in the visual processing regions in schizophrenia. The concomitant reduction of MTR and FA that occurs in a circumscribed region of the occipito-temporal WM suggests abnormalities in the architecture of myelinated axons in schizophrenia. The WM integrity in the occipito-temporal regions indexed by the FA, rather than the MTR, predicted PS deficit in patients.

A specific strength of our approach is the use of 7T MTI technology that has a higher sensitivity to detect macromolecular pathology. Although the exchange rate is independent of field strength (Henkelman et al, 1993), MT imaging at high-field MRI benefits from the increased SNR, and prolongation of water $\mathrm{T} 1$ relaxation time, such that the MT effect can be detected before the partially saturated longitudinal magnetization of free water pool returns to equilibrium (Mougin et al, 2010). Furthermore, at high field, chemical shift between exchange site proton and water proton increases, allowing greater exchange rate to be used without approaching the fast exchange rate limit. A large chemical shift helps to reduce or even avoid the direct saturation of free water in addition to reducing the macromolecular water MT background (Ward et al, 2000).

Our observation of occipito-temporal MTR reduction in schizophrenia adds to the existing MTI studies in patients with established illness (for a tabulated review, see the Supplementary Material S1). The earliest MTR study in 
schizophrenia used lobar regions of interest covering entire brain and reported prominent temporal MTR reduction (Foong et al, 2000). Most recently, Bachmann et al (2011), using an imputed voxelwise analysis, demonstrated that the most significant MTR reduction in schizophrenia occurs in the calcarine, lingual, fusiform gyrus, and inferior temporal cortex. Nevertheless, there are several studies that observe localized MTR changes in other brain regions, such as frontal lobe and the insula (Supplementary Material S2). Methodological differences such as scanner strength and analytical approach could explain some of these discrepancies. For example, a significant increase in MTR in patients has only been reported in tract-based ROI studies (Mandl et al, 2010; de Weijer et al, 2011), and has not been observed in voxelwise analyses. Tract-based ROI studies have reported increased MTR confined to the uncinate and arcuate fasiculi, and have so far not investigated whole brain differences (Mandl et al, 2010; de Weijer et al, 2011), making it difficult to ascertain whether large sized effects with reduced MTR were present in other brain regions. Further, frontal MTR defects are most often seen in first episode samples (Bagary et al, 2003; Bohner et al, 2012; Price et al, 2010). It is possible that frontal myelination defects in schizophrenia diminish with the progression of illness or respond to antipsychotics. Some support to the latter view comes from a series of studies evaluating the WM effects of atypical antipsychotics (Bartzokis et al, 2012), leading to the notion that some interventions may have a selective effect on ameliorating WM abnormalities in schizophrenia.

Schizophrenia is likely to be associated with a distributed abnormality in myelination (Davis et al, 2003). If this is indeed true, a more prominent reduction in myelin content in patients is expected in cortical regions that are relatively heavily myelinated. Interestingly, whole brain myelin mapping suggests both occipital and inferior temporal/ parahippocampal regions are relatively rich in myelin content compared with higher-order association cortices in normal subjects (Glasser and Van Essen, 2011). In the present study, a combined FA and MTR reduction was located in the WM regions that have a high probability of including occipito-temporal fibers (ILF) and occipitofrontal fibers (inferior fronto-occipital fasciculus). Several studies have previously reported reduced WM integrity of ILF in schizophrenia (Ellison-Wright and Bullmore, 2009). ILF appears to originate from extrastriate visual association regions and project to parahippocampal region and limbic regions such as the amygdala (Catani et al, 2003). Our results suggest that abnormal myelination of the fibers projecting to and from visual association regions are present despite treatment with antipsychotics in clinically stable patients. This is consistent with studies that observe reduced myelin-related gene expression in occipital cortex (Matthews et al, 2012), and adds to a growing body of evidence implicating visual processing defects in schizophrenia (Javitt, 2009).

PS deficit that persists despite symptomatic remission is an important indicator of chronic disability and poor functional outcome in schizophrenia (Nuechterlein et al, 2011). To date, the nature of relationship between reduced WM integrity and PS deficit remains unclear in schizophrenia despite observations of such relationships for other cognitive domains such as verbal memory (Szeszko et al, 2008). None of the previous MTR studies in schizophrenia have sought the relationship between cognitive dysfunction and MTR changes (Supplementary Material S1). Our results suggest that the integrity of connections emerging from visual association regions is likely to influence the PS performance in patients. FA, rather than the MTR of the affected regions, predicted PS performance. This dissociation has been noted in other studies as well (Schiavone et al, 2009). This suggests that a disruption of the axonal architecture in occipito-temporal regions showing reduced myelin content is likely to be crucial for the expression of impaired PS, a key cognitive deficit in schizophrenia.

The absence of a significant relationship between FA of the occipito-temporal region and PS in healthy controls suggests that occipito-temporal FA is unlikely to contribute to the interindividual variability in the PS performance in the current sample of healthy controls. FA of specific tracts such as superior longitudinal fasciculus not investigated in the present study may account for the variability in PS performance in controls (Turken et al, 2008). Further, in healthy individuals, age contributes to a substantial portion of the observed relationship in the variance between WM integrity and PS (Madden et al, 2012). Most of the WM integrity-cognition relationships in healthy controls is notable only after middle age, where the interindividual variation in both cognitive ability and the WM integrity becomes more pronounced(Burgmans et al, 2011). Further, this age-related FA-PS association appears to be driven by overall WM integrity across different tracts, rather than being limited to a single WM pathway (Penke et al, 2010; Vernooij, 2009). Unlike previous studies that showed a relationship between PS performance and FA in healthy controls (Kohama et al, 2012; Penke et al, 2010; Voineskos et al, 2012b), our control group included healthy younger adults (age mean $(\mathrm{SD})=32(8.2)$ years) in whom large agerelated differences in WM integrity are unlikely to be present.

Several shortcomings must be considered when interpreting the present observations. Our sample size is modest, although comparable to several published MTR studies. Although MTI provides a quantitative measure of regional myelin content, MTR values are affected by regional freewater concentration. As a result, a reduction in MTR may be a result of local tissue edema or partial volume effect that reduces macromolecular concentration (Stikov et al, 2011). We used an advanced tissue segmentation procedure that reduces the probability of partial volume effect (Ashburner, 2007). The use of voxelwise approach rather than tractography approach ensured an unbiased localization of whole brain changes of MTR in our sample, but the observed FA/ MTR changes may not apply for the entire course of WM fasciculi. As most subjects in our sample are men, caution is required when generalizing these results to women. In this sample, we evaluated PS as a cognitive variable, but did not evaluate other cognitive domains. Given the previous observations linking verbal memory to FA reductions (Szeszko et al, 2008), extensive assessment of cognitive function is warranted in future DTI/MTI studies. Further, visual processing deficits have been proposed to be a key phenotypic feature that defines a subset of patients with more severe cognitive impairment (Butler et al, 2005). 
Examining occipito-temporal myelin integrity alongside extended cognitive and functional assessments of this feature can provide further insights into the pathophysiology.

In summary, using combined WM imaging in schizophrenia, we have shown a significant reduction in the myelin content in the occipito-temporal WM. Our results indicate that aberrant conduction along the WM tracts from visual processing regions to a higher-order association cortices may contribute to PS defects in patients. The combined WM imaging approach is likely to be a sensitive tool to quantify neurobiological correlates of persisting cognitive defects in patients with schizophrenia.

\section{ACKNOWLEDGEMENTS}

We gratefully acknowledge all the volunteers who participated in this study. We also thank Dr Vijender Balain for the recruitment of patients, Kathleen Shaw, and Kay Head for their assistance in image acquisition. We are grateful for the support received from the Medical Research Council (G0601442) that helped us to develop and optimize the image acquisition protocol. L Palaniyappan is supported by a research training fellowship from the Wellcome Trust. This work was funded by an internal grant from the School of Community Health Sciences, University of Nottingham.

\section{DISCLOSURE}

L Palaniyappan is supported by Wellcome Trust fellowship; has received a Young Investigator Travel Fellowship from Eli Lilly in 2011; has received income from SPMM Ltd for providing MRCPsych revision courses and has received book royalties from the Oxford University Press. O Mougin and A Al-Radaideh received income from the respective primary employers. PA Gowland has institutional grant support from the Medical Research Council and has received research funding from Idec Ltd, Proctor and Gamble Unilever Norgine. PF Liddle has received honoraria for academic presentations from GlaxoSmithKline, AstraZeneca, Janssen-Cilag, Bristol Myers Squibb and Eli Lilly; has taken part in advisory panels for Bristol Myers Squibb, Eli Lilly, Pfizer and GlaxoSmithKline; has received institutional grant support from the Medical Research Council (G0601442 and MR/J01186X/1) and the Dr Hadwen Trust; and receives book royalties from the Royal College of Psychiatrists. The remaining authors declare no conflict of interest.

\section{REFERENCES}

American Psychiatric Association (1994). Diagnostic and Statistical Manual of Mental Disorders, Fourth Edition. APA: Washington, DC.

Ammons RB, Ammons CH (1962). The Quick Test (QT): provisional manual. Psychol Rep 11: 111-161.

Annett M (1970). A classification of hand preference by association analysis. Br J Psychol 61: 303-321.

Ashburner J (2007). A fast diffeomorphic image registration algorithm. Neuroimage 38: 95-113.

Bachmann S, Haffer S, Beschoner P, Viviani R (2011). Imputation techniques for the detection of microstructural changes in schizophrenia, with an application to magnetization transfer imaging. Schizophr Res 132: 91-96.

Bagary MS, Symms MR, Barker GJ, Mutsatsa SH, Joyce EM, Ron MA (2003). Gray and white matter brain abnormalities in firstepisode schizophrenia inferred from magnetization transfer imaging. Arch Gen Psychiatry 60: 779-788.

Bartzokis G, Lu PH, Raven EP, Amar CP, Detore NR, Couvrette AJ et al (2012). Impact on intracortical myelination trajectory of long acting injection versus oral risperidone in first-episode schizophrenia. Schizophr Res 140: 122-128.

Bartzokis G, Lu PH, Tingus K, Mendez MF, Richard A, Peters DG et al (2010). Lifespan trajectory of myelin integrity and maximum motor speed. Neurobiol Aging 31: 1554-1562.

Bohner G, Milakara D, Witthaus H, Gallinat J, Scheel M, Juckel G et al (2012). MTR abnormalities in subjects at ultra-high risk for schizophrenia and first-episode schizophrenic patients compared to healthy controls. Schizophr Res 137: 85-90.

Burgmans S, EHBM Gronenschild, Fandakova Y, Shing YL, van, Vuurman EFPM Boxtel MPJ et al (2011). Age differences in speed of processing are partially mediated by differences in axonal integrity. NeuroImage 55: 1287-1297.

Butler PD, Zemon V, Schechter I, Saperstein AM, Hoptman MJ, Lim KO et al (2005). Early-stage visual processing and cortical amplification deficits in schizophrenia. Arch Gen Psychiatry 62: 495-504.

Catani M, Jones DK, Donato R, Ffytche DH (2003). Occipitotemporal connections in the human brain. Brain 126: 2093-2107.

Chen JT, Kuhlmann T, Jansen GH, Collins D., Atkins HL, Freedman MS et al (2007). Voxel-based analysis of the evolution of magnetization transfer ratio to quantify remyelination and demyelination with histopathological validation in a multiple sclerosis lesion. NeuroImage 36: 1152-1158.

Davis KL, Stewart DG, Friedman JI, Buchsbaum M, Harvey PD, Hof PR et al (2003). White matter changes in schizophrenia: evidence for myelin-related dysfunction. Arch Gen Psychiatry 60: 443-456.

Ellison-Wright I, Bullmore E (2009). Meta-analysis of diffusion tensor imaging studies in schizophrenia. Schizophr Res 108: 3-10.

Foong J, Maier M, Barker GJ, Brocklehurst S, Miller DH, Ron MA (2000). In vivo investigation of white matter pathology in schizophrenia with magnetisation transfer imaging. J Neurol Neurosurg Psychiatr 68: 70-74.

Ge Y, Grossman RI, Udupa JK, Babb JS, Kolson DL, McGowan JC (2001). Magnetization transfer ratio histogram analysis of gray matter in relapsing-remitting multiple sclerosis. AJNR Am J Neuroradiol 22: 470-475.

Glasser MF, Van Essen DC (2011). Mapping human cortical areas in vivo based on myelin content as revealed by T1- and T2weighted MRI. J Neurosci 31: 11597-11616.

Henkelman RM, Huang X, Xiang Q-S, Stanisz GJ, Swanson SD, Bronskill MJ (1993). Quantitative interpretation of magnetization transfer. Magn Reson Med 29: 759-766.

Javitt DC (2009). Sensory processing in schizophrenia: neither simple nor intact. Schizophr Bull 35: 1059-1064.

Kohama SG, Rosene DL, Sherman LS (2012). Age-related changes in human and non-human primate white matter: from myelination disturbances to cognitive decline. Age (Dordr) 34: 1093-1110.

Kubicki M, Park H, Westin CF, Nestor PG, Mulkern RV, Maier SE et al (2005). DTI and MTR abnormalities in schizophrenia: analysis of white matter integrity. Neuroimage 26: 1109-1118.

Leckman JF, Sholomskas D, Thompson D, Belanger A, Weissman MM (1982). Best estimate of lifetime psychiatric diagnosis: a methodological study. Arch Gen Psychiatry 39: 879-883.

Liddle PF, Ngan ETC, Duffield G, Kho K, Warren AJ (2002). Signs and Symptoms of Psychotic Illness (SSPI): a rating scale. $\mathrm{Br} \mathrm{J}$ Psychiatry 180: 45-50. 
Madden DJ, Bennett IJ, Burzynska A, Potter GG, Chen N, Song AW (2012). Diffusion tensor imaging of cerebral white matter integrity in cognitive aging. Biochim Biophys Acta 1822: 386-400.

Mandl RCW, Schnack HG, Luigjes J, Heuvel MP, van den, Cahn W, Kahn RS et al (2010). Tract-based analysis of magnetization transfer ratio and diffusion tensor imaging of the frontal and frontotemporal connections in schizophrenia. Schizophr Bull 36: 778-787.

Matthews PR, Eastwood SL, Harrison PJ (2012). Reduced myelin basic protein and actin-related gene expression in visual cortex in schizophrenia. PLoS ONE 7: e38211.

Mori S, Wakana S, Nagae-Poetscher LM, van Zijl PC (2005). MRI atlas of human white matter. Elsevier: Amsterdam, The Netherlands.

Mougin OE, Coxon RC, Pitiot A, Gowland PA (2010). Magnetization transfer phenomenon in the human brain at 7T. NeuroImage 49: 272-281.

Nuechterlein KH, Subotnik KL, Green MF, Ventura J, Asarnow RF, Gitlin MJ et al (2011). Neurocognitive predictors of work outcome in recent-onset schizophrenia. Schizophr Bull 37: S33-S40.

Penke L, Maniega SM, Murray C, Gow AJ, Valdes Hernandez MC, Clayden JD et al (2010). A general factor of brain white matter integrity predicts information processing speed in healthy older people. J Neurosci 30: 7569-7574.

Pérez-Iglesias R, Tordesillas-Gutiérrez D, McGuire PK, Barker GJ, Roiz-Santiañez R, Mata I et al (2010). White matter integrity and cognitive impairment in first-episode psychosis. Am J Psychiatry 167: 451-458.

Polders D, Hoogduin H, Donahue MJ, Henrikse J, Luijten P (2009). Comparison of SNR and diffusion parameters on 1.5, 3.0 and 7.0 Tesla. Proc ISMRM 2009; Honolulu 1406.

Price G, Cercignani M, Chu EM, Barnes TRE, Barker GJ, Joyce EM et al (2010). Brain pathology in first-episode psychosis: magnetization transfer imaging provides additional information to MRI measurements of volume loss. Neuroimage 49: 185-192.

Schiavone F, Charlton RA, Barrick TR, Morris RG, Markus HS (2009). Imaging age-related cognitive decline: a comparison of diffusion tensor and magnetization transfer MRI. J Magn Reson Imaging 29: 23-30.

Schmierer K, Scaravilli F, Altmann DR, Barker GJ, Miller DH (2004). Magnetization transfer ratio and myelin in postmortem multiple sclerosis brain. Ann Neurol 56: 407-415.

Schmierer K, Wheeler-Kingshott CAM, Boulby PA, Scaravilli F, Altmann DR, Barker GJ et al (2007). Diffusion tensor imaging of post mortem multiple sclerosis brain. Neuroimage 35: 467-477.

Speck O, Zhong K (2009). Diffusion tensor imaging at 7T: expectations vs. reality check. Proc ISMRM 2009; Honolulu 1462.

Stikov N, Perry LM, Mezer A, Rykhlevskaia E, Wandell BA, Pauly JM et al (2011). Bound pool fractions complement diffusion measures to describe white matter micro and macrostructure. Neuroimage 54: 1112-1121.

Szeszko PR, Robinson DG, Ashtari M, Vogel J, Betensky J, Sevy S et al (2008). Clinical and neuropsychological correlates of white matter abnormalities in recent onset schizophrenia. Neuropsychopharmacology 33: 976-984.

Turken AU, Whitfield-Gabrieli S, Bammer R, Baldo JV, Dronkers NF, Gabrieli JDE (2008). Cognitive processing speed and the structure of white matter pathways: Convergent evidence from normal variation and lesion studies. NeuroImage 42: 1032-1044.

Tzourio-Mazoyer N, Landeau B, Papathanassiou D, Crivello F, Etard O, Delcroix N et al (2002). Automated anatomical labeling of activations in SPM using a macroscopic anatomical parcellation of the MNI MRI single-subject brain. Neuroimage 15: 273-289.

Vernooij MW IM (2009). WHite matter microstructural integrity and cognitive function in a general elderly population. Arch Gen Psychiatry 66: $545-553$.

Voineskos AN, Felsky D, Kovacevic N, Tiwari AK, Zai C, Chakravarty MM et al (2012a). Oligodendrocyte genes, white matter tract integrity, and cognition in schizophrenia. Cereb Cortex; e-pub ahead of print, doi:10.1093/cercor/bhs188.

Voineskos AN, Rajji TK, Lobaugh NJ, Miranda D, Shenton ME, Kennedy JL et al (2012b). Age-related decline in white matter tract integrity and cognitive performance: A DTI tractography and structural equation modeling study. Neurobiol Aging 33: 21-34.

Walterfang M, Wood SJ, Velakoulis D, Pantelis C (2006). Neuropathological, neurogenetic and neuroimaging evidence for white matter pathology in schizophrenia. Neurosci Biobehav Rev 30: 918-948.

Ward KM, Aletras AH, Balaban RS (2000). A new class of contrast agents for MRI based on proton chemical exchange dependent saturation transfer (CEST). J Magn Reson 143: 79-87.

Wechsler D (1997). WAIS-III: Administration and Scoring Manual. Harcourt Brace \& Company: San Diego, CA.

Weijer AD, de, Neggers SFW, KMS Diederen, RCW Mandl, Kahn RS, Hulshoff Pol HE et al (2011). Aberrations in the arcuate fasciculus are associated with auditory verbal hallucinations in psychotic and in non-psychotic individuals. Hum Brain Mapp 34: 626-634.

Whitford TJ, Ford JM, Mathalon DH, Kubicki M, Shenton ME (2012). Schizophrenia, myelination, and delayed corollary discharges: a hypothesis. Schizophr Bull 38: 486-494.

WHO Collaborating Centre for Drug Statistics and Methodology (2003): Guidelines for ATC Classification and DDD Assignment.

Zahr NM, Rohlfing T, Pfefferbaum A, Sullivan EV (2009). Problem solving, working memory, and motor correlates of association and commissural fiber bundles in normal aging: a quantitative fiber tracking study. Neuroimage 44: 1050-1062.

Supplementary Information accompanies the paper on the Neuropsychopharmacology website (http://www.nature.com/npp) 